\title{
ARTICLE
}

\section{Structure-based assortment of herbal analogues against spike protein to restrict COVID-19 entry through hACE2 receptor: An in-silico approach}

\author{
Sourav Santra ${ }^{1 \#, ~ S a s t i ~ G o p a l ~ D a s ~}{ }^{1 \#}$, Suman Kumar Halder ${ }^{2}$, Kuntal Ghosh³, Amrita \\ Banerjee $^{4}$, Amiya Kumar Panda ${ }^{1,5}$ and Keshab Chandra Mondal1,2* \\ 1BIF Center, Vidyasagar University, Midnapore-721102, West Bengal, India \\ ${ }^{2}$ Department of Microbiology, Vidyasagar University, Midnapore-721102, West Bengal, India \\ ${ }^{3}$ Department of Biological Sciences, Midnapore City College, Midnapore-721129, West Bengal, India \\ ${ }^{4}$ Department of Biotechnology, Oriental Institute of Science \& Technology, Midnapore-721102, West Bengal, India \\ ${ }^{5}$ Department of Chemistry and Chemical Technology, Vidyasagar University, Midnapore-721102, West Bengal, India
}

\begin{abstract}
On-going global pandemic COVID-19 has spread all over the world and has led to more than 1.97 million deaths till date. Natural compounds may be useful to protecting health in this perilous condition. Mechanism of shuttle entry of SARS-COV-2 virus is by interaction with viral spike protein with human angiotensin-converting enzyme-2 (ACE-2) receptor. To explore potential natural therapeutics, 213 important phytochemicals of nine medicinal plants Aconitum heterophyllum, Cassia angustifolia, Cymbopogon flexuosus, Cymbopogon martinii, Nux vomica, Phyllanthus urinaria, Swertia chirayita, Justicia adhatoda, Vetiveria zizanioides were selected for in-silico molecular docking against the spike protein of SARS-COV-2 and compared with recently prescribed drug chloroquine, ramdesivir, lopinavir and hydroxychloroquine. Results revealed that rhamnocitrin of $P$. urinaria, 1,5-dihydroxy-3,8-dimethoxyxanthone of $S$. chirayita and laevojunenol of $V$. zizanioides potentially binds with the receptor binding site of SARS-COV-2 spike glycoprotein and more robustly destabilized the RBD-ACE-2 binding over chloroquine, ramdesivir, lopinavir and hydroxychloroquine. It was also found that laevojunenol, rhamnocitrin, and 1,5-dihydroxy-3,8-dimethoxyxanthone qualified the criteria for drug-likeness as per Lipinski rule. After attachment of the selected phytochemical with the spike protein the affinity of the later towards ACE-2 was minimized and the effect of 1,5-dihydroxy-3,8dimethoxyxanthone and laevojunenol was superior. Hence, rhamnocitrin of $P$. urinaria, 1,5-dihydroxy-3,8-dimethoxyxanthone of $S$. chirayita and laevojunenol of $V$. zizanioides, are potential therapeutic molecules for SARS-COV-2, which upon binding with spike protein changes the affinity of the spike towards ACE- 2 and therefore restrict the entry of the virus into a human cell. Subsequent clinical validation is needed to confirm these phytochemicals as drugs to combat COVID-19.

Acta Biol Szeged 64(2):159-171 (2020)
\end{abstract}

\section{KEY WORDS}

angiotensin-converting-enzyme-2 COVID-19

medicinal plants

molecular docking

spike glycoprotein

\section{INTRODUCTION}

Presently, the world's population is in under tremendous pressure, due to COVID-19 pandemic. Worldwide, more than 90 million people are infected and among them more than 1.97 million people died till date (as per WHO). Deaths are increasing by leaps and bounds due to community transmission and the scientists are deliberately trying to find drugs from natural and synthetic origin to use as a potential antiviral agent. The entry of the COVID-19 virus into human cells is mediated via transmembrane spike (S) glycoprotein that contributes to the cell receptor binding, tissue tropism, and pathogenesis. Spike protein has conserved motifs have three domains namely S1, S2 and N. The S1-domain has a conserved receptor-binding domain (RBD), which recognizes the angiotensin-converting enzyme-2 (ACE-2) receptor (Bourgonje et al. 2019; Yao et al. 2020) which is the initial step of entry mechanism into the host cells (Walls et al. 2020; Wang et al. 2020). The expression of ACE-2 is higher in the intestinal epithelium and pulmonary pneumocytes than other tissues. The interaction of $S$ protein and ACE-2 results in imbalance of the renin-angiotensin system in the lungs as well as immunological intolerance, which leads to acute lung injury such as pulmonary oedema (Yao et al. 2020; Yang et al. 2007; Kuba et al. 2005). The entry of coronavirus into susceptible cells is a complex process that requires the concerted action of receptor binding and proteolytic processing of the S protein,

\# Both the authors contributed equally. 
which endorses virus-cell fusion (Walls et al. 2020). In recent time, chloroquine, hydroxychloroquine, remdesivir, lopinavir, arbidol are drugs of choice for COVID treatment but they have limitations and side effects (Wang et al. 2020; Cao et al. 2007; Rismanbaf et al. 2020; Yazdany et al. 2020). Under this emergency, several conventional and non-conventional medicines are being tested around the world to restrict viral transmission and to develop effective therapeutics. In this perspective, medicinal plants especially those employed in traditional medicine for the treatments of virus-allied symptoms have recently come under scientific surveillance as they contain bioactive compounds that could be useful for development of potential drugs against COVID like viral diseases.

Since ancient human civilization, many phytochemicals of diversified unique properties are being explored for customized treatments of variety of diseases owing to their analgesic, antipyretic, antioxidative, anticancer, immunomodulatory, anti-inflammatory, antimicrobial, anti-carcinogenic and many other notable properties (Jaiswal and Williams 2017; Suresh and Abraham 2020; Koparde et al. 2019). In global perspective, Lion's share of the world population still depends on plant derived products to formulate drugs to treat their health problems. Some selective herbal products have low cytotoxicity and high bioavailability and are effective for the treatment of different viral diseases (Divya et al. 2020). The potential biological roles of plant's secondary metabolites have now been explored in the blockage of virus particle's entry, their multiplication and pathogenesis (ul Qamar et al. 2020). Among the different plants, P. urinaria (commonly called gripe weed) have potential ethnomedicinal importance against asthma, bronchitis, cough, tuberculosis, fever, influenza, digestive pain, conjunctivitis and anaemia. Besides, this plant also effective against hepatitis A-C, herpes and HIV (Singh 2018). S. chirayita (also is known as chirayta) is useful for the cure of different diseases and containing anti-inflammatory, antioxidant and antiviral compounds. S. chirayita has also very good antiviral activity against herpes and papilloma virus (Singh 2018; Kumar and Van Staden 2016). Likewise, V. zizanioides (vetivergrass; Hindi: Khas-Khas) used to treat many skin and nervous disorders and claimed to inhibit the dengue NS2B-NS3 virus (Lavanya et al. 2016). A. heterophyllum also helps in the treatment of common cold, flu, and malaria bronchitis, persistent cough and upper respiratory tract infections (Paramanick et al. 2017). C. flexuosus commonly used against headaches, diabetes, rheumatism, hypertension, wounds, fever and bone fractures (Rajeswara Rao 2013). Nux vomica is used against colds and flu, particularly in the early stages of any virus infection (Singh 2018). These all plants are selected based on their ethno-botanical importance and the study
Table 1. Synthetic compounds and their binding energy with SARSCOV-2 spike glycoprotein.

\begin{tabular}{lll}
\hline SI No. & Compound names & Binding energy (kcal/mol) \\
\hline 1 & Ramdesivir & -8.1 \\
2 & Lopinavir & -11.8 \\
3 & Chloroquine & -6.7 \\
4 & Hydroxychloroquine & -6.6 \\
\hline
\end{tabular}

of literature. The present study was aimed to explore the interaction of natural compounds of A. heterophyllum, $C$. angustifolia, C. flexuosus, C. martinii, N. vomica, P. urinaria, S. chirayita, J. adhatoda, V. zizanioides with the spike protein of the SARS-CoV-2 virus. The 3D structure of S protein was constructed and its binding ability against the 213 phytochemicals of the above-mentioned medicinal plants were evaluated. The post binding effect of the conjugated spike protein with ACE-2 was also addressed in order to explore the effectiveness of natural compounds as potential anti-COVID drug.

\section{MATERIALS AND METHODS}

\section{Retrieval of protein sequence and prediction of homolo- gous structure}

The reference spike glycoprotein YP_009724390.1 sequence of human corona virus SARS-COV-2 collected from NCBI. Three-dimensional structure of corona viral spike glycoprotein (PDB ID: 6VSB) and human Angiotensin Converting Enzyme-2 (ACE-2) (PDB ID: 4APH) were retrieved from RCSB Protein Data Bank (https:// www.rcsb.org/) in PDB format and modelling of the spike glycoprotein (YP_009724390.1) was carried out in SWISS-MODEL server (Waterhouse et al. 2018) and further analysed by using PyMol (DeLano 2002). Quality assessment of this spike glycoprotein model was validated by PROCHECK by analysing the Ramachandran plot (Laskowski et al. 1993).

\section{Retrieval of ligands structure}

Three-dimensional structure of 213 natural compounds of A. heterophyllum, C. angustifolia, C.flexuosus, C. martinii, $N$. vomica, P. urinaria, S. chirayita, J. adhatoda, V. zizanioides and drugs remdesivir, lopinavir, chloroquine and hydroxychloroquine was retrieved from Indian Medicinal Plants, Phytochemistry and Therapeutics (IMPPAT) database (Mohanraj et al. 2018), PubChem (Kim et al. 2016) on the basis of literature survey and listed in Table 1-3.

\section{Protein-ligand docking}

Prior docking, the water molecules were removed from the 
Table 2. List of natural plant derived compounds whose binding energy is higher than -7 kcal/mol when binds with SARS-COV-2 spike glycoprotein.

\begin{tabular}{|c|c|c|c|c|}
\hline No & Compound names & Plant species & Binding energy (kcal/mol) & H-bond interaction \\
\hline 1 & Hetidine & A. heterophyllum & -9.7 & 371 \\
\hline 2 & Isoatisine & A. heterophyllum & -8.7 & - \\
\hline 3 & Hetisinone & A. heterophyllum & -8.6 & 370,489 \\
\hline 4 & Veratridine & A. heterophyllum & -8.5 & 115,167 \\
\hline 5 & 6-benzoylheteratisine & A. heterophyllum & -8.4 & 377,379 \\
\hline 6 & Hetisine & A. heterophyllum & -8.4 & 370 \\
\hline 7 & Atidine & A. heterophyllum & -8.3 & $377,379,457$ \\
\hline 8 & Beta-carotene & A. heterophyllum & -8.3 & - \\
\hline 9 & Atisine & A. heterophyllum & -8.2 & 455 \\
\hline 10 & Dihydroatisine & A. heterophyllum & -8.1 & 338,339 \\
\hline 11 & Aricine & A. heterophyllum & -8 & 370,457 \\
\hline 12 & Benzoylmesaconine & A. heterophyllum & -8 & 488 \\
\hline 13 & Lactone atisenol & A. heterophyllum & -8 & 457 \\
\hline 14 & Lappaconitine & A. heterophyllum & -7.6 & $357,473,475$ \\
\hline 15 & 6-acetylheteratisine & A. heterophyllum & -7.4 & 379 \\
\hline 16 & Heterophyllisine & A. heterophyllum & -7.4 & 489 \\
\hline 17 & Aconitine & A. heterophyllum & -7.3 & - \\
\hline 18 & Anisoylaconine & A. heterophyllum & -7.3 & $377,457,473$ \\
\hline 19 & Hypaconitine & A. heterophyllum & -7.3 & 371,373 \\
\hline 20 & Mesaconitine & A. heterophyllum & -7.3 & 371,373 \\
\hline 21 & Phytosterols & A. heterophyllum & -7.3 & 457 \\
\hline 22 & Jesaconitine & A. heterophyllum & -7.2 & 165,355 \\
\hline 23 & Isorhamnetin 3-gentiobioside & C. angustifolia & -8.4 & $369,417,457,487,489,493$ \\
\hline 24 & Kaempferol & C. angustifolia & -8.1 & 457,477 \\
\hline 25 & Aloe-emodin & C. angustifolia & -7.7 & 457,477 \\
\hline 26 & Tinnevellinglucoside & C. angustifolia & -7.6 & $371,403,409,505$ \\
\hline 27 & Sennaglucosides & C. angustifolia & -9.8 & $343,370,371,453,476,478,493$ \\
\hline 28 & Emodin-8-glucoside & C. angustifolia & -8.9 & $369,371,375,376,405,406,409$ \\
\hline 29 & Rhein & C. angustifolia & -8.6 & - \\
\hline 30 & Isorhamnetin & C. angustifolia & -8.6 & 457,477 \\
\hline 31 & Arundoin & C. flexuosus & -8.7 & - \\
\hline 32 & Phytosterols & C. flexuosus & -7.8 & 457 \\
\hline 33 & Humulene & C. flexuosus & -7.5 & 457 \\
\hline 34 & Caryophylene oxide & C. martinii & -7 & 478 \\
\hline 35 & Stryvomicine A & N. vomica & -9.2 & - \\
\hline 36 & Beta-colubrine chloromethochloride & N. vomica & -8.8 & 457,477 \\
\hline 37 & Alpha-colubrine chloromethochloride & N. vomica & -8.5 & \\
\hline 38 & Oleanolic acid & P. urinaria & -8.7 & 370,457 \\
\hline 39 & Trans-8,9-Dihydro-benz(a)anthracene-8,9-diol & P. urinaria & -8.7 & - \\
\hline 40 & Corilagin & P. urinaria & -8.7 & $379,457,487,493$ \\
\hline 41 & Naringin & P. urinaria & -8.7 & $343,375,403,405,437$ \\
\hline 42 & Furosin & P. urinaria & -8.7 & $417,456,457,493,494$ \\
\hline 43 & Kaempferol 7-methyl ether 4'-glucoside & P. urinaria & -8.7 & $417,456,457,493,494$ \\
\hline 44 & Gallocatechingallate & P. urinaria & -8.6 & 457 \\
\hline 45 & Ellagic acid & P. urinaria & -8.5 & $371,405,408,409$ \\
\hline 46 & Cleistanthol & P. urinaria & -8.4 & - \\
\hline 47 & Quercetin & P. urinaria & -8.4 & $371,455,457,477$ \\
\hline 48 & Daucosterol & P. urinaria & -8.4 & 377,488 \\
\hline 49 & Spruceanol & P. urinaria & -8.3 & 457,490 \\
\hline 50 & Quercitrin & P. urinaria & -8.3 & 457,488 \\
\hline
\end{tabular}


Santra et al.

Table 2. Continued.

\begin{tabular}{|c|c|c|c|c|}
\hline No & Compound names & Plant species & Binding energy (kcal/mol) & H-bond interaction \\
\hline 51 & $\begin{array}{l}\text { 13-Methyl-6,7,8,9,11,12,14,15,16,17- } \\
\text { decahydrocyclopenta[a]phenanthrene-3,17-diol }\end{array}$ & P. urinaria & -8.2 & - \\
\hline 52 & Betulinic acid & P. urinaria & -8.2 & $408,456,457$ \\
\hline 53 & Epigallocatechingallate & P. urinaria & -8.2 & $369,379,457,487,490$ \\
\hline 54 & Rutin & P. urinaria & -8.2 & $\begin{array}{l}372,374,375,403,405,437,439, \\
453,505\end{array}$ \\
\hline 55 & Epicatechin-3-gallate & P. urinaria & -8.1 & $381,417,457,487,489$ \\
\hline 56 & Beta-sitosterol & P. urinaria & -8.1 & - \\
\hline 57 & Glochidiol & P. urinaria & -8 & 408 \\
\hline 58 & Epicatechin & P. urinaria & -7.9 & $379,381,487,489$ \\
\hline 59 & Epigallocatechin & P. urinaria & -7.9 & $379,381,487,489$ \\
\hline 60 & Betulin & P. urinaria & -7.9 & 456,457 \\
\hline 61 & Rhamnocitrin & P. urinaria & -7.9 & $379,487,489$ \\
\hline 62 & Brevifolincarboxylic acid & P. urinaria & -7.8 & $375,377,415$ \\
\hline 63 & Ethyl brevifolincarboxylate & P. urinaria & -7.5 & $371,457,477$ \\
\hline 64 & Digallic acid & P. urinaria & -7.4 & $370,457,477,478$ \\
\hline 65 & Methyl brevifolincarboxylate & P. urinaria & -7.3 & $455,457,490$ \\
\hline 66 & Urinatetralin & P. urinaria & -7.1 & 381 \\
\hline 67 & Episwertenol & S. chirayita & -9.7 & - \\
\hline 68 & Hopenol B & S. chirayita & -9.3 & - \\
\hline 69 & Erythrodiol & S. chirayita & -9.1 & - \\
\hline 70 & Friedlein & S. chirayita & -9.1 & 357 \\
\hline 71 & Chiratenol & S. chirayita & -8.9 & - \\
\hline 72 & Kairatenol & S. chirayita & -8.7 & - \\
\hline 73 & Swertanone & S. chirayita & -8.7 & - \\
\hline 74 & Oleanolic acid & S. chirayita & -8.6 & - \\
\hline 75 & Taraxasterol acetate & S. chirayita & -8.5 & 378,408 \\
\hline 76 & Swertenol & S. chirayita & -8.3 & 466 \\
\hline 77 & Amarogentin & S. chirayita & -8.2 & $370,371,372,457,490$ \\
\hline 78 & Swertiapuniside & S. chirayita & -8.1 & $372,403,439,505,506$ \\
\hline 79 & Ursolic acid & S. chirayita & -8.1 & 457 \\
\hline 80 & 1,8-Dihydroxy-2,6-dimethoxy-9H-xanthen-9-one & S. chirayita & -7.9 & - \\
\hline 81 & Mangiferin & S. chirayita & -7.9 & $379,456,457,492$ \\
\hline 82 & 1,5-dihydroxy-3,8-dimethoxyxanthone & S. chirayita & -7.7 & $406,409,417$ \\
\hline 83 & Swertianin & S. chirayita & -7.6 & $415,377,369$ \\
\hline 84 & Decussatin & S. chirayita & -7.5 & 457,477 \\
\hline 85 & Demethylbellidifolin & S. chirayita & -7.5 & 409 \\
\hline 86 & Isobellidifolin & S. chirayita & -7.5 & 371,457 \\
\hline 87 & Swerchirin & S. chirayita & -7.5 & 371,457 \\
\hline 88 & 7,11-Epoxy-eremophila-1,9-dien-8-a-ol & V. zizanioides & -8.2 & 457 \\
\hline 89 & Eudesmane & V. zizanioides & -8 & - \\
\hline 90 & Cadalene & V. zizanioides & -7.9 & - \\
\hline 91 & Khusene & V. zizanioides & -7.9 & - \\
\hline 92 & 10-epi-Acora-3,11-dien-15-al & V. zizanioides & -7.8 & 457,478 \\
\hline 93 & Beta-vetivone & V. zizanioides & -7.8 & 457 \\
\hline 94 & 15-nor-prezizaan-7-one & V. zizanioides & -7.7 & 457,478 \\
\hline 95 & Isokhusimol & V. zizanioides & -7.7 & 457 \\
\hline 96 & Isovalencenol & V. zizanioides & -7.7 & 457,477 \\
\hline 97 & Khusiol & V. zizanioides & -7.7 & - \\
\hline 98 & $\begin{array}{l}(1 \mathrm{~S}, 2 \mathrm{~S}, 8 \mathrm{R})-2,6,7,7 \text {-tetramethyltricyclo[6.2.1.01,5] } \\
\text { undecane }\end{array}$ & V. zizanioides & -7.7 & - \\
\hline
\end{tabular}


Table 2. Continued.

\begin{tabular}{|c|c|c|c|c|}
\hline No & Compound names & Plant species & Binding energy (kcal/mol) & $\mathrm{H}$-bond interaction \\
\hline 99 & Acora-2,4-diene & V. zizanioides & -7.6 & - \\
\hline 100 & Beta-cadinene & V. zizanioides & -7.6 & - \\
\hline 101 & Beta-vetivenene & V. zizanioides & -7.6 & - \\
\hline 102 & Khusimone & V. zizanioides & -7.6 & - \\
\hline 103 & Khusinol oxide & V. zizanioides & -7.6 & 478 \\
\hline 104 & 7,15-epoxyprezizaane & V. zizanioides & -7.5 & 370 \\
\hline 105 & 10-epi-Acor-3-en-5-one & V. zizanioides & -7.5 & - \\
\hline 106 & Allo-khusiol & V. zizanioides & -7.5 & 370 \\
\hline 107 & Alpha-vetispirene & V. zizanioides & -7.5 & - \\
\hline 108 & Eremophilane & V. zizanioides & -7.5 & - \\
\hline 109 & Khusilal & V. zizanioides & -7.5 & 457 \\
\hline 110 & Khusinodiol & V. zizanioides & -7.5 & 457 \\
\hline 111 & Khusitone & V. zizanioides & -7.5 & - \\
\hline 112 & 13-nor-Eudesm-5-en-11-one & V. zizanioides & -7.4 & 457 \\
\hline 113 & Cedrane & V. zizanioides & -7.4 & - \\
\hline 114 & Epizizanal & V. zizanioides & -7.4 & - \\
\hline 115 & Isovetiselinenol & V. zizanioides & -7.4 & - \\
\hline 116 & Khusinol & V. zizanioides & -7.4 & 490 \\
\hline 117 & Laevojunenol & V. zizanioides & -7.4 & 489 \\
\hline 118 & 11,12,13-tri-nor-cis-Eudesm-5-en-7-one & V. zizanioides & -7.3 & - \\
\hline 119 & Beta-Vetispirene & V. zizanioides & -7.3 & - \\
\hline 120 & Isokhusenic acid & V. zizanioides & -7.3 & - \\
\hline 121 & Isokhusinol oxide & V. zizanioides & -7.3 & 489 \\
\hline 122 & Nootkatone & V. zizanioides & -7.3 & - \\
\hline 123 & Cadin-4-en-10-ol & V. zizanioides & -7.2 & - \\
\hline 124 & Khusimol & V. zizanioides & -7.2 & - \\
\hline 125 & Ac1lb1ow & V. zizanioides & -7.1 & - \\
\hline 126 & Alpha-vetivone & V. zizanioides & -7.1 & - \\
\hline 127 & Cyclocopacamphenol & V. zizanioides & -7.1 & 457,477 \\
\hline 128 & Epizizanone & V. zizanioides & -7.1 & - \\
\hline 129 & Khusol & V. zizanioides & -7.1 & 370 \\
\hline
\end{tabular}

three-dimensional structure of the spike glycoprotein. The molecular docking study was performed for exploration of the binding affinity of the spike glycoprotein with the 213 selected natural phytochemicals in addition with remdesivir, lopinavir, chloroquine and hydroxychloroquine through AutoDock Vina [version 1.1.2] (Trott and Olson 2010). SARS-COV-2 spike protein S1 domain chains (A, $\mathrm{B}, \mathrm{C})$ have common reference active site of K417, G446, Y449, F486, N487, Y489, Q493, Q498, T500, N501, G502, Y505 (Walls et al. 2020; Wang et al. 2020; Yan et al. 2020; Lan et al. 2020). These sites were initially targeted for grid based molecular docking study with the selected natural phytochemicals. The grid box dimensions were $68 \AA \times 90 \AA \times 58 \AA$ with a spacing of $1 \AA$ and center set at coordinate $219.172,224.276$ and 287.134 in $\mathrm{x}, \mathrm{y}$, and $\mathrm{z}$ axis, respectively, centring around the ACE-2 binding domain. An array of ligands was screened based on binding energy at active site amino acid residues, and subsequently blindly docked with SARS-COV-2 spike protein using AutoDock with new grid dimension to cover the whole spike glycoprotein. The grid coordinate considered as 126 $\AA \times 126 \AA \times 126 \AA$ with a spacing of $1 \AA$ and center set to coordinate 211.921, 226.209 and 247.631 in the $x, y$ and $\mathrm{z}$ axis with 24 exhaustiveness, respectively. During blind docking, the size of grids was kept at maximum covering the whole surface of the protein to allow the ligand to bind in an unbiased binding pocket. Polar $\mathrm{H}$ charges of the Gasteiger-type were assigned to the receptor molecule and torsions were detected. Default settings of AutoDock Vina were used for docking studies. 
Santra et al.

Table 3. List of natural plant derived compounds whose binding energy is less than $-7 \mathrm{kcal} / \mathrm{mol}$ when binds with SARS-COV-2 spike glycoprotein.

\begin{tabular}{|c|c|c|c|}
\hline No & Compound names & Plant species & Binding energy (kcal/mol) \\
\hline 1 & Phytosterols & A. heterophyllum & -6.9 \\
\hline 2 & Delphatines & A. heterophyllum & -6.7 \\
\hline 3 & Lycoctonine & A. heterophyllum & -6.6 \\
\hline 4 & DI-borneol & C. flexuosus & -6 \\
\hline 5 & Citronellal & C. flexuosus & -5.7 \\
\hline 6 & Citral & C. flexuosus & -5.7 \\
\hline 7 & Methyleugenol & C. flexuosus & -5.6 \\
\hline 8 & Myrcene & C. flexuosus & -5.1 \\
\hline 9 & 6-Methylhept-5-en-2-ol & C. flexuosus & -5 \\
\hline 10 & Triacontane & C. flexuosus & -4.9 \\
\hline 11 & Decanal & C. flexuosus & -4.8 \\
\hline 12 & Trans-2-Hepten-1-ol & C. flexuosus & -4.5 \\
\hline 13 & Beta-caryophyllene & C. martinii & -6.6 \\
\hline 14 & 3-carene & C. martinii & -6.5 \\
\hline 15 & Carvylacetate & C. martinii & -6.4 \\
\hline 16 & P-cymene & C. martinii & -6.3 \\
\hline 17 & Alpha-terpineol & C. martinii & -6.3 \\
\hline 18 & Dihydrocarvone & C. martinii & -6.3 \\
\hline 19 & (-)-3-carene & C. martinii & -6.3 \\
\hline 20 & Beta-terpineol & C. martinii & -6.2 \\
\hline 21 & Perillyl alcohol & C. martinii & -6.2 \\
\hline 22 & D-carvone & C. martinii & -6.2 \\
\hline 23 & (-)-cis-carveol & C. martinii & -6.1 \\
\hline 24 & Cis,cis-farnesol & C. martinii & -6.1 \\
\hline 25 & Alpha-farnesene & C. martinii & -6.1 \\
\hline 26 & Limonene & C. martinii & -5.9 \\
\hline 27 & Eucalyptol & C. martinii & -5.8 \\
\hline 28 & Dihydrocarveol & C. martinii & -5.7 \\
\hline 29 & Geranyl acetate & C. martinii & -5.6 \\
\hline 30 & Geraniol & C. martinii & -5.3 \\
\hline 31 & 6-Methyl-5-hepten-2-one & C. martinii & -5.2 \\
\hline 32 & 6-Octen-1-ol, 3,7-dimethyl-, (R)- & C. martinii & -5.2 \\
\hline 33 & Geranyl butyrate & C. martinii & -5.1 \\
\hline 34 & (-)-Linalool & C. martinii & -4.9 \\
\hline 35 & 2-Nonanol & C. martinii & -4.7 \\
\hline 36 & 1,5-Hexadiyne & J. adhatoda & -5.4 \\
\hline 37 & 2-(2,5-Hexadiynyloxy)tetrahydro-2H-pyran & J. adhatoda & -5.2 \\
\hline 38 & Heptasiloxane, 1,1,3,3,5,5,7,7,9,9,11,11,13,13-tetradecamethyl- & J. adhatoda & -5.1 \\
\hline 39 & Hexasiloxane,1,1,3,3,5,5,7,7,9,9,11,11-dodecamethyl- & J. adhatoda & -4.7 \\
\hline 40 & Octasiloxane, $1,1,3,3,5,5,7,7,9,9,11,11,13,13,15,15$-hexadecamethyl- & J. adhatoda & -4.3 \\
\hline 41 & Pentasiloxane, 1,1,3,3,5,5,7,7,9,9-decamethyl- & J. adhatoda & -4.3 \\
\hline 42 & Nirtetralin & P. urinaria & -6.9 \\
\hline 43 & Phyltetralin & P. urinaria & -6.9 \\
\hline 44 & Lintetralin & P. urinaria & -6.8 \\
\hline 45 & Hypophyllanthin & P. urinaria & -6.7 \\
\hline 46 & Syringin & P. urinaria & -6.7 \\
\hline 47 & Virgatusin & P. urinaria & -6.7 \\
\hline 48 & Dehydrochebulic acid trimethyl ester & P. urinaria & -6.6 \\
\hline 49 & Ferulic acid & P. urinaria & -6.5 \\
\hline 50 & 5-demethoxyniranthin & P. urinaria & -6.5 \\
\hline
\end{tabular}


Table 3. Continued.

\begin{tabular}{|c|c|c|c|}
\hline No & Compound names & Plant species & Binding energy (kcal/mol) \\
\hline 51 & Cucurbic acid & P. urinaria & -6.3 \\
\hline 52 & Methyl gallate & P. urinaria & -6.2 \\
\hline 53 & Niranthin & P. urinaria & -6.2 \\
\hline 54 & 4-O-Methylgallic acid & P. urinaria & -6 \\
\hline 55 & Phyllanthin & P. urinaria & -5.9 \\
\hline 56 & (6r)-menthiafolic acid & P. urinaria & -5.9 \\
\hline 57 & 4-hydroxybenzaldehyde & P. urinaria & -5.7 \\
\hline 58 & 5-hydroxymethylfurfural & P. urinaria & -4.6 \\
\hline 59 & Dl-tryptophan & S. chirayita & -6.9 \\
\hline 60 & Gentianine & S. chirayita & -6.6 \\
\hline 61 & Enicoflavine & S. chirayita & -5.5 \\
\hline 62 & Dl-arginine & S. chirayita & -5.2 \\
\hline 63 & Nonacosyl_hentriacontanoate & S. chirayita & -5 \\
\hline 64 & Octadecanoate & S. chirayita & -4.8 \\
\hline 65 & Glutamate & S. chirayita & -4.2 \\
\hline 66 & Amorphane & V. zizanioides & -7 \\
\hline 67 & 6,12-Epoxy-elema-1,3-diene & V. zizanioides & -6.9 \\
\hline 68 & 13-nor-4,5-Epoxyeudesm-6-en-11-one & V. zizanioides & -6.9 \\
\hline 69 & Bisabolane & V. zizanioides & -6.7 \\
\hline 70 & 3-carene & V. zizanioides & -6.5 \\
\hline 71 & 15-nor-Funebran-3-one & V. zizanioides & -6.5 \\
\hline 72 & Beta-pinene & V. zizanioides & -6.5 \\
\hline 73 & Cis-Isoeugenol & V. zizanioides & -6.5 \\
\hline 74 & Cyclocopacamphan-12-al & V. zizanioides & -6.5 \\
\hline 75 & Isobisabolene & V. zizanioides & -6.5 \\
\hline 76 & Nootkatol & V. zizanioides & -6.4 \\
\hline 77 & Alpha-terpineol & V. zizanioides & -6.3 \\
\hline 78 & 2-Methoxy-4-vinylphenol & V. zizanioides & -5.8 \\
\hline 79 & 4-vinylphenol & V. zizanioides & -5.8 \\
\hline 80 & Eucalyptol & V. zizanioides & -5.8 \\
\hline 81 & Eugenol & V. zizanioides & -5.6 \\
\hline 82 & O-cresol & V. zizanioides & -5.5 \\
\hline 83 & M-cresol & V. zizanioides & -5.4 \\
\hline 84 & Oleamide & V. zizanioides & -5 \\
\hline
\end{tabular}

\section{Lipinski rule for validation of drug-likeness}

Lipinski's rule of 5 helps to find out drug likeness of any experimental compound. Five rules are (i) molecular mass of the drug should be less than 500 Dalton, (ii) high lipophilicity (expressed as LogP less than 5), (iii) less than 5 hydrogen bond donors, (iv) less than 10 hydrogen bond acceptors, (v) molar refractivity should be between 40-130. The predictable drugs sites Lipinski Rule were checked by using SCFBio web-based database (Lipinski 2004).

\section{Protein-protein docking}

Spike glycoprotein (in free form and complex with selected phytochemicals) (Table 4) and ACE-2 were considered for protein-protein docking with receptor-binding domain
(RBD) of the spike glycoprotein (S1 subunit) using HDOCK server (Yan et al. 2020). Precisely, the 19-41 amino acid resides of ACE-2 was employed for the docking with 417505 amino acids of spike glycoprotein $\mathrm{S} 1$ subunit. In total, four ACE-2 docking experiments were performed with spike glycoprotein as per specification stated in Table 4.

\section{RESULTS AND DISCUSSION}

\section{Homologous structure prediction of spike glycopro- tein and its validation}

The study of molecular docking and the ligand-based 


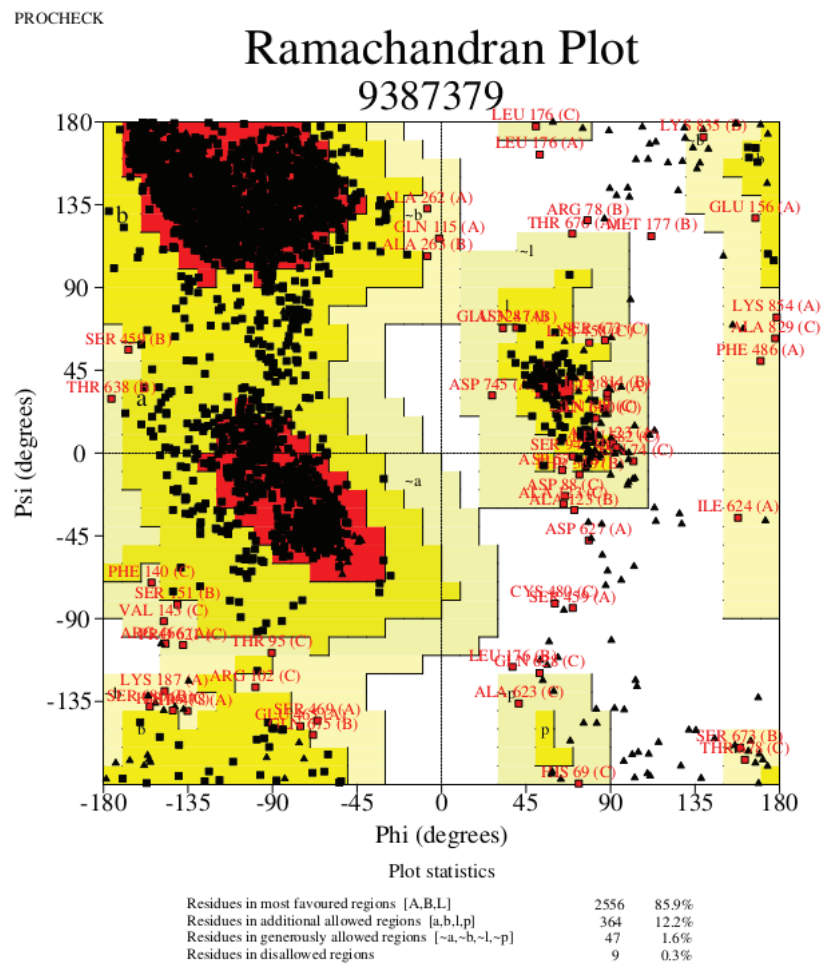

Figure 1. Ramachandran plot of COVID-19 SARS-CoV-2 spike glycoprotein.

computer-aided drug discovery approach involves energy, affinity and three-dimensional structural conformationsbased analysis of ligands interaction with the target of interest. The binding ability of a ligand molecule to a specific target site depends on occurrence of proper cleft, proper hydrogen bonding and the nature of residues present at the target site and their interactive energy balance (Tripet et al. 2004). The binding affinity of a ligand for a targeted receptor is measured by binding energy. Lower binding energy implies that binding affinity is high whereas higher energy endorses the reverse (Zhou et al. 2020). In the present study, three-dimensional (3D) homologous structure of spike glycoprotein was generated from the reference protein sequence (YP_009724390.1) using the cryo-EM structure (6VSB) through SWISSMODEL server and further analyzed through PyMol.
PyMol is a molecular visualization tool; used here to align the predicted three-dimensional structure of the spike glycoprotein of SARS-CoV-2 into the cryo-EM structure of 6VSB. The Root-mean-square deviation (RMSD) of this model was computed as $0.278 \AA$. Further, the predicted 3 D structure was validated through RAMACHANDRAN plot using PROCHECK. The result revealed that 85.9\% belongs to the most favourable region, $12.2 \%$ in allowed region, $1.6 \%$ in the generously allowed region, and only $0.3 \%$ in disallowed region (Fig. 1). Though cryo EM structure of SARS-COV-2 spike glycoprotein (6VSB) was available, some amino acids were not resolved properly at different locations due to its $3.46 \AA$ resolution. To get the complete structure of spike, homology modelling was performed. Good quality structure with $98.1 \%$ residues at ordered form indicated the proper conformational packaging of protein.

\section{Assessment of binding affinity of 213 ligands with spike protein and their subsequent screening}

A spike glycoprotein of SARS-COV-2 has three domains namely S1, S2 and N. The S2 domain intercedes the membrane fusion process and the S1 domain utilizes human angiotensin-converting enzyme-2 (hACE-2) as the receptor to infect human cells (Pandey et al. 2020). The literature review revealed that the receptor-binding domain (RBD) of the S1 subunit of spike protein binds with the target cell ACE-2 receptor and forms the RBDACE-2 complex. According to recent report, ACE-2 could mediate SARS-CoV-2 binding by spike protein key residues of K417, G446, Y449, F486, N487, Y489, Q493, Q498, T500, N501, G502, Y505 (Walls et al. 2020; Wang et al. 2020; Yan et al. 2020; Lan et al. 2020). In this in-silico study, we attempted to explore the binding affinity of 213 phytochemicals in addition to remdesivir, lopinavir, chloroquine and hydroxychloroquine with the active site residues of the spike glycoprotein (Fig. 2A). The selection of the natural ligands of plant origin was primarily made on the basis of (i) minimal binding energy ( $<-7 \mathrm{kcal} / \mathrm{mol})$ and (ii) formation of at least one $\mathrm{H}$-bond with the active site residues (417-505) in the S1 subunit of the spike glycoprotein. These criteria were fulfilled by 23 compounds which were further blindly docked with whole spike glycoprotein

Table 4. Different complex of spike glycoprotein (free and ligand bound) and ACE2 docking in HDOCK and their binding score.

\begin{tabular}{lll}
\hline Molecular docking complex & HDOCK score & RMSD value \\
\hline Spike glycoprotein dock with ACE2 & -360.86 & 0.51 \\
Spike glycoprotein and rhamnocitrin dock with ACE2 & -360.86 & 0.51 \\
Spike glycoprotein and 1,5-dihydroxy-3,8-dimethoxyxanthone dock with ACE2 & -243.15 & 481.28 \\
Spike glycoprotein and laevojunenol dock with ACE2 & -238.13 & 480.02 \\
\hline
\end{tabular}


Table 5. Physicochemical properties of natural plant derived compounds and their binding energy during molecular docking. Different capital alphabets before amino acids indicate different polypeptide chains.

\begin{tabular}{llllcr}
\hline Compound name & Amino acids at docked sites & \multicolumn{2}{c}{ Lipinski criteria for drug-likeness } \\
\cline { 3 - 5 } & & MW (g/mol) & $\begin{array}{l}\text { Hydrogen } \\
\text { bond donor }\end{array}$ & $\begin{array}{l}\text { Hydrogen } \\
\text { bond acceptor }\end{array}$ & XLogP3-AA $\begin{array}{l}\text { Molar } \\
\text { refractivity }\end{array}$ \\
\hline Rhamnocitrin & $\begin{array}{l}\text { B/PHE-490, B/SER-477, } \\
\text { C/SER-371, B/ARG-457, }\end{array}$ & 300.26 & 3 & 6 & 2.2 \\
1,5-dihydroxy-3,8-dimethoxyxanthone & $\begin{array}{l}\text { B/GLU-406, B/GLN-409, } \\
\text { B/LYS-417 }\end{array}$ & 288.26 & 2 & 6 & 2.37 \\
Laevojunenol & B/TYR-489 & 222.37 & 1 & 1 & 77.145782 \\
\hline
\end{tabular}

(Table 1-2) to analyze the affinity of selected molecules at the active site of spike glycoprotein (417-505) instead of other cleft and pockets. It was evident that rhamnocitrin of $P$. urinaria, 1,5-dihydroxy-3,8-dimethoxyxanthone of S. chirayita, laevojunenol and khusinol of $V$. zizanioides are capable to bind with active site residues of the S1 subunit of spike glycoprotein (S) in 0 (zero) RMSD pose (Fig. 2). So, these three phytochemicals are the best ligand molecules for spike protein active site which restricts smooth interaction between spike and ACE-2.

The molecular docking study of spike protein with remdesivir revealed that this drug has the capability to bind with S1 domain through H-bond with the ARG403, ASP405 and ARG408 of B and PHE374, SER375 and TYR508 of C chain with binding energy of $-8.1 \mathrm{kcal} /$ mol. However, though remdesivir binds with the spike protein at S1 domain, but the site of attachment is not the active site of spike protein. Lopinavir interacts with the S2 subunit through amino acid residues GLN957, THR961 and associated with binding energy of -11.8 $\mathrm{kcal} / \mathrm{mol}$. Alongside, it was also revealed that chloroquine and hydroxychloroquine binds with LEU455, GLY485, PHE490,PRO491 and PRO559, PHE855, THR573, ILE587 residues of S2 domain having the binding energy of -6.7 and $-6.3 \mathrm{kcal} / \mathrm{mol}$, respectively (Table 3 ). It is evident that among the four drugs neither one is capable to bind at active site of spike glycoprotein.

The docking results were analysed based on a combination of binding energy, clustering score, shape complementarity, functional significance of the binding pocket and favourable interactions including $\mathrm{H}$ bonds.

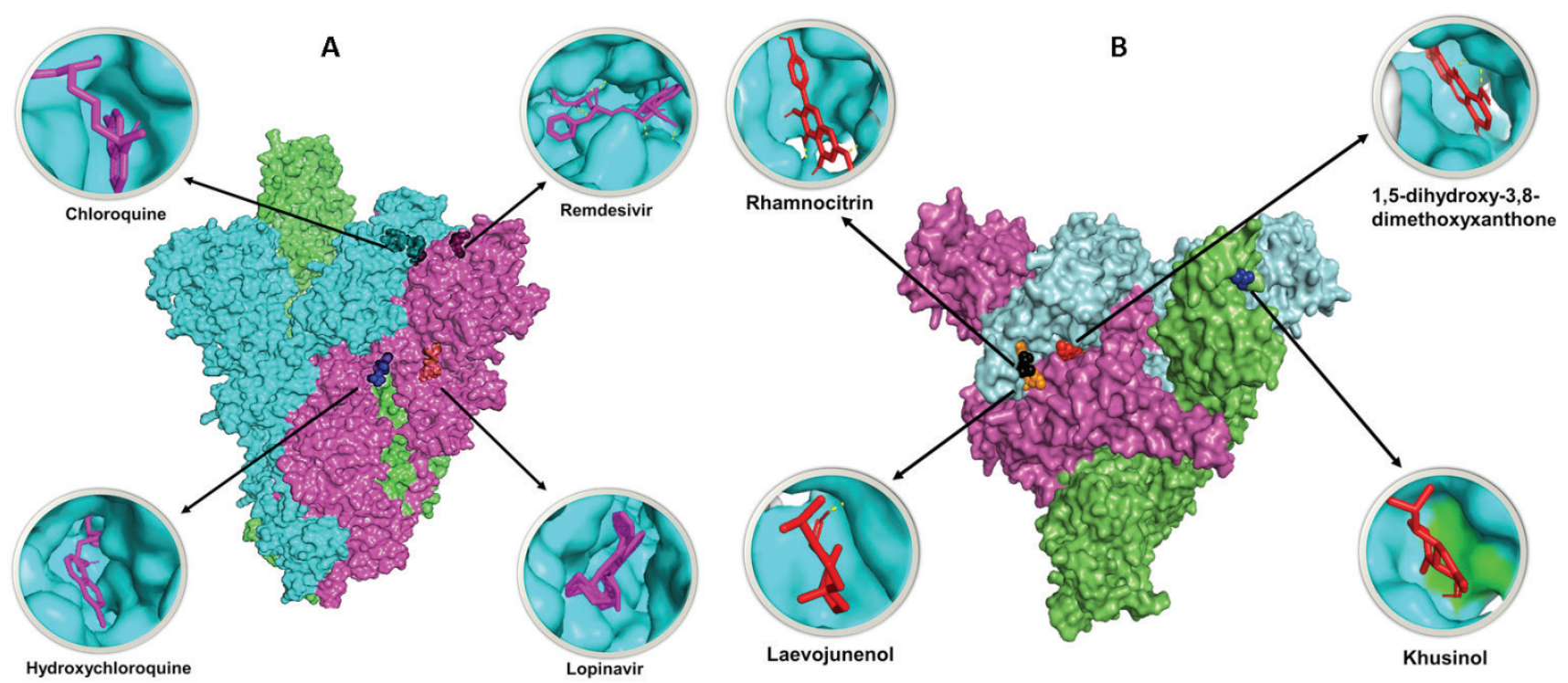

Figure 2. Binding of (A) remdesivir, lopinavir, chloroquine and hydroxychloroquine and (B) rhamnocitrin, 1,5-dihydroxy-3,8-dimethoxyxanthone, laevojunenol and khusinol with SARS-CoV-2 spike glycoprotein. 


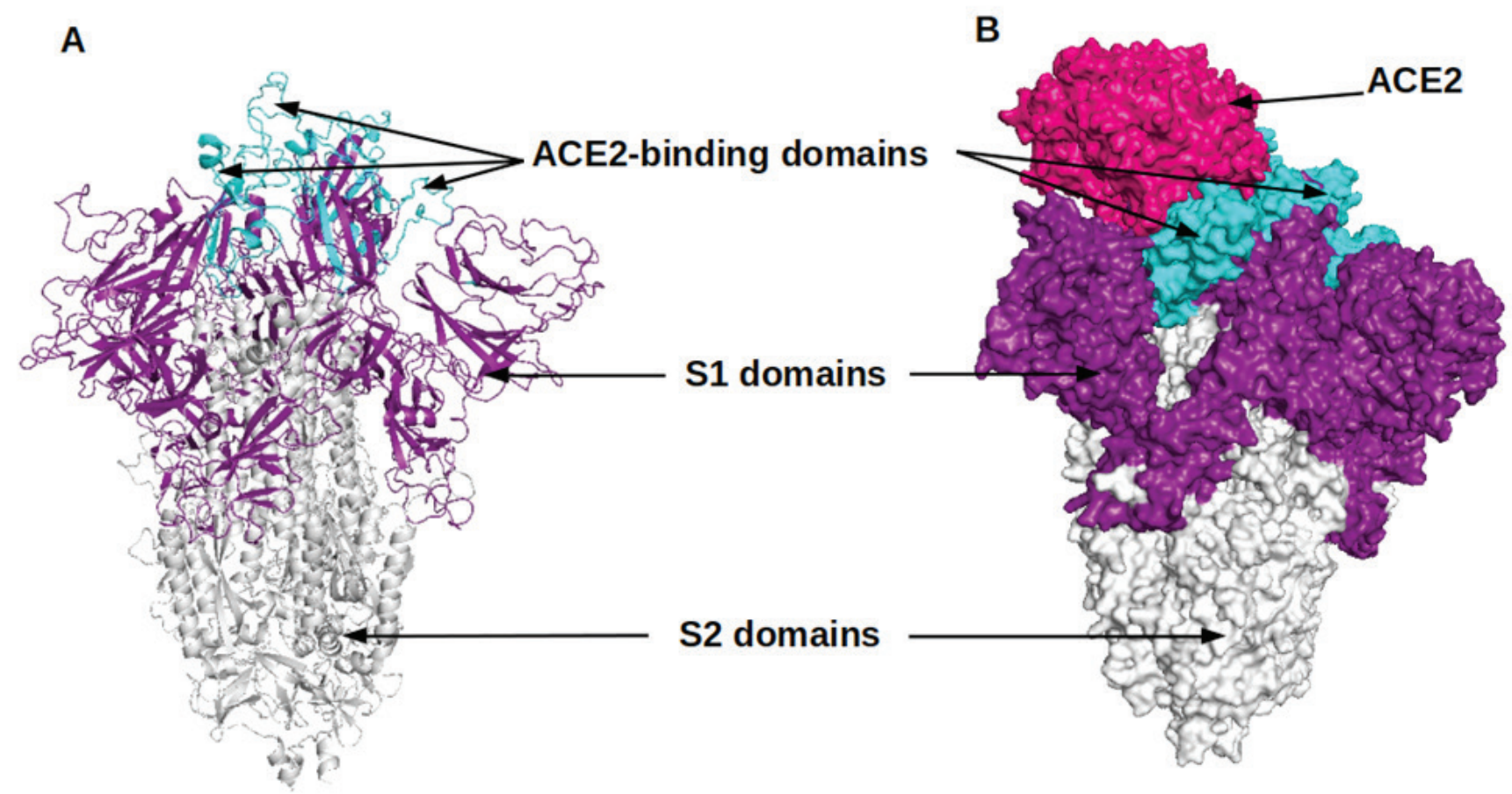

Figure 3. Binding of hACE2 with SARS-CoV-2 spike glycoprotein. (A) cartoon view and (B) surface view.

\section{Validation of drug-likeness}

Lipinski rule of five is a rule of thumb to check the drug's likeness of any chemical compound. It acts as a filter to screen potential therapeutic agents/drugs just at the initiation of the program, thereby minimizing the labour and costs of clinical drug development and to a large extent prevents late-stage clinical failures (Raj et al. 2019; Pandey et al. 2020). In this study, three selected phytochemicals were examined for their drug-likeness in the light of the rules (Table 5). The results clearly demonstrated that rhamnocitrin, 1,5-dihydroxy-3,8 dimethoxyxanthone and laevojunenol qualified the rule.

\section{Ligand binding effect analysis on spike glycoprotein- ACE-2 interaction}

Finally, the selected drugs were individually used to study effective inhibition of RBD-ACE2 complex formation. The interaction of spike glycoprotein with ACE-2 is depicted in Fig. 3. In order to verify the possible effect of the ligands in spike-ACE-2 interaction, the S glycoprotein-ligand docked complexes were further docked with ACE-2 protein in HDOCK web server. HDOCK is a web serverbased protein-protein and protein-DNA/RNA docking tool. This web server based molecular docking revealed that the binding energy of spike protein-ACE-2 interaction was -360.86 and rmsd value 0.51 which decreased to -243.15 and 481.28 rmsd for 1,5-dihydroxy-3,8-dimethoxyxanthone, -238.13 and 480.02 for laevojunenol,
-360.86 and 0.51 for rhamnocitrin separately pre-fixed with spike glycoprotein, respectively. Apart from the effect on binding energy, it was also evident that the binding of 1,5-dihydroxy-3,8-dimethoxyxanthone and laevojunenol triggers the shifting of interaction sites of both the partners from their active sites which may hamper the viral entry into human cell (Fig. 4). Also 1,5-dihydroxy-3,8-dimethoxyxanthone of S. chirayita, laevojunenol of $V$. zizanioides binding pose being poor, these compounds can be considered as potential inhibitor of S-glycoprotein and human ACE-2 interaction.

\section{CONCLUSION}

In conclusion, we can state that the present computer-aided in-silico study of exploration of preventive drugs against COVID-19 revealed that natural herbal phytochemicals like rhamnocitrin; 1,5-dihydroxy-3,8-dimethoxyxanthone and laevojunenol of $P$. urinaria, $S$. chirayita, and $V$. zizanioides have immense potential to restrict the onset of SARSCOV-2 disease due to their ability to interrupt the normal viral spike protein and ACE-2 interaction upon binding to the spike protein. The potential of 1,5-dihydroxy-3,8dimethoxyxanthone and laevojunenol was proved to be superior. Maybe these compounds will be useful as potential preventive drugs, however, further experiments are necessary to validate their effects. 

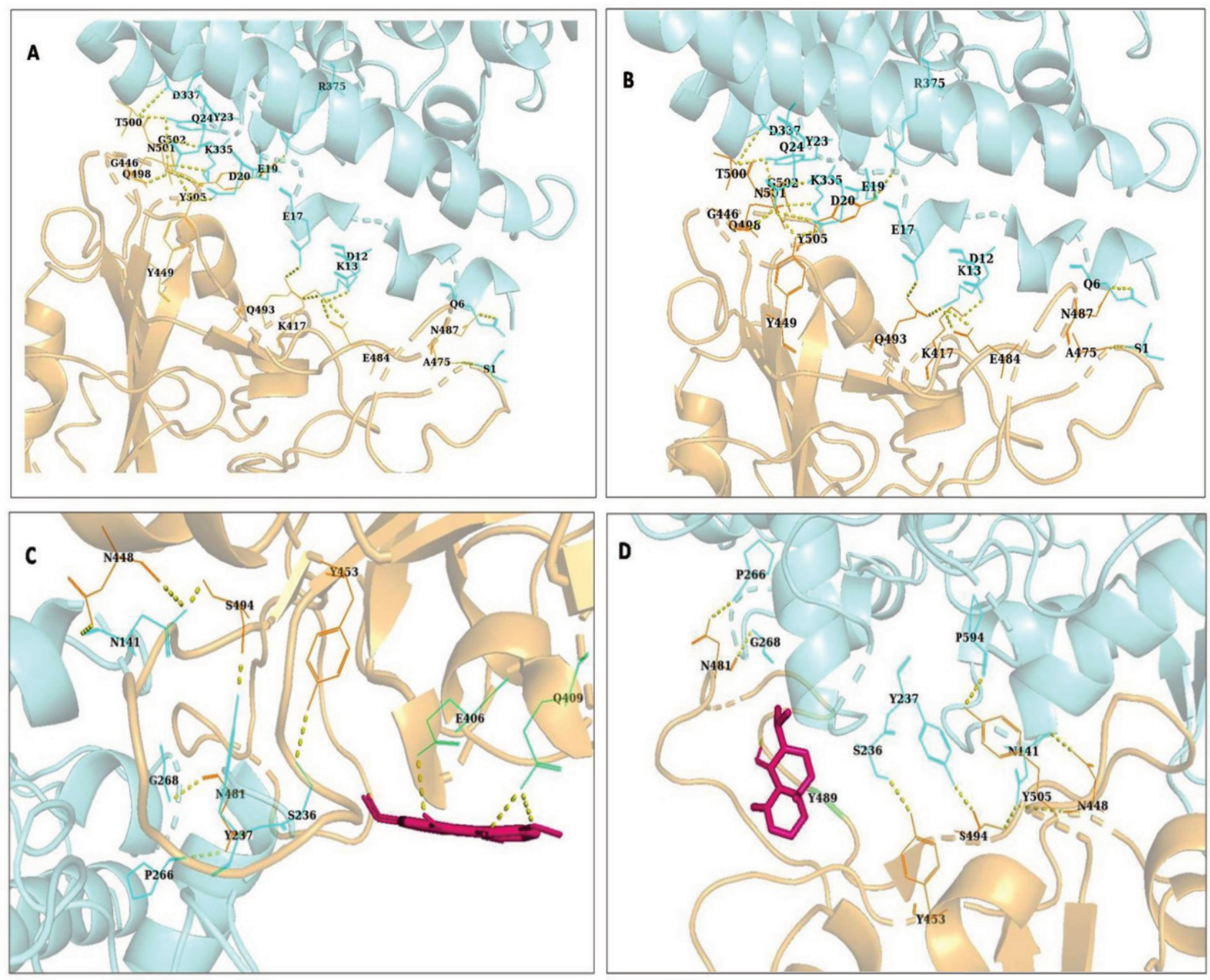

Figure 4. Spike protein bound with ACE2 in open condition (A). Binding of hACE2 with SARS-CoV-2 spike glycoprotein already bound 1,5-dihydroxy-3,8-dimethoxyxanthone (C) and laevojunenol (D). Change in binding position between hACE2 with SARS-CoV-2 spike glycoprotein was apparent in case of all except rhamnocitrin (B).

\section{Acknowledgement}

We would like to thank DBT, Government of India for financial assistant for BIF Center, Vidyasagar University, Midnapore-721102, West Bengal, India.

\section{References}

Bourgonje AR, Abdulle AE, Timens W, Hillebrands JL, Navis GJ, Gordijn SJ, Bolling MC, Dijkstra G, Voors AA, Osterhaus AD, van der Voort PH (2020) Angiotensinconverting enzyme-2 (ACE2), SARS-CoV-2 and pathophysiology of coronavirus disease 2019 (COVID-19). J Pathol 251(3):228-248.
Cao B, Wang Y, Wen D, Liu W, Wang J, Fan G, Ruan L, Song B, Cai Y, Wei M, Li X (2020) A trial of lopinavir-ritonavir in adults hospitalized with severe Covid-19. N Engl J Med 382:1787-1799.

DeLano WL (2002) Pymol: An open-source molecular graphics tool. CCP4 Newslett Prot Crystallogr 40(1):82-92.

Dev S (1999) Ancient-modern concordance in Ayurvedic plants: some examples. Environ Health Perspect 107(10):783-789.

Divya M, Vijayakumar S, Chen J, Vaseeharan B, Durán-Lara EF (2020) A review of South Indian medicinal plant has the ability to combat against deadly viruses along with COVID-19? Microb Pathog 104277.

Jaiswal YS, Williams LL (2017) A glimpse of Ayurveda-The forgotten history and principles of Indian traditional 
medicine. J Tradit Compl Med 7(1):50-53.

Kim S, Thiessen PA, Bolton EE, Chen J, Fu G, Gindulyte A, Han L, He J, He S, Shoemaker BA, Wang J (2016) PubChem substance and compound databases. Nucleic Acids Res 44:D1202-D1213.

Koparde AA, Doijad RC, Magdum CS (2019) Natural products in drug discovery. In Pharmacognosy-Medicinal Plants. IntechOpen.

Kuba K, Imai Y, Rao S, Gao H, Guo F, Guan B, Huan Y, Yang P, Zhang Y, Deng W, Bao L (2005) A crucial role of angiotensin converting enzyme 2 (ACE2) in SARS coronavirus-induced lung injury. Nat Med 11(8):875-879.

Kumar V, Van Staden J. (2015) A review of Swertia chirayita (Gentianaceae) as a traditional medicinal plant. Front Pharmacol 6:308.

Lan J, Ge J, Yu J, Shan S, Zhou H, Fan S, Zhang Q, Shi X, Wang Q, Zhang L, Wang X. (2020) Structure of the SARS-CoV-2 spike receptor-binding domain bound to the ACE2 receptor. Nature 581(7807):215-220.

Laskowski RA, MacArthur MW, Moss DS, Thornton JM (1993) PROCHECK: a program to check the stereochemical quality of protein structures. J Appl Cryst 26(2):283-291.

Lipinski CA. (2004) Lead-and drug-like compounds: the rule-of-five revolution. Drug Discov Today Technol 1(4):337-341.

Lavanya P, Ramaiah S, Anbarasu A (2016) Ethyl 4-(4-methylphenyl)-4-pentenoate from Vetiveriazizanioides inhibits dengue NS2B-NS3 protease and prevents viral assembly: a computational molecular dynamics and docking study. Cell Biochem Biophys 74:337-351.

Mohanraj K, Karthikeyan BS, Vivek-Ananth RP, Chand RB, Aparna SR, Mangalapandi P, Samal A (2018) IMPPAT: A curated database of Indian Medicinal Plants, Phytochemistry and Therapeutics. Sci Rep 8(1):4329.

Pandey P, Rane JS, Chatterjee A, Kumar A, Khan R, Prakash A, Ray S (2020) Targeting SARS-CoV-2 spike protein of COVID-19 with naturally occurring phytochemicals: an in-silico study for drug development. J Biomol Struct Dyn 22:1-11.

Paramanick D, Panday R, Shukla SS, Sharma V (2017) Primary pharmacological and other important findings on the medicinal plant "Aconitum heterophyllum" (aruna). J Pharmacopunct 20(2):89-92.

Raj S, Sasidharan S, Dubey VK, Saudagar P (2019) Identification of lead molecules against potential drug target protein MAPK4 from L. donovani: An in-silico approach using docking, molecular dynamics and binding free energy calculation. PloS One 14(8):e0221331.

Rajeswara Rao BR (2013) Biological activities and medicinal uses of the essential oil and extracts of lemongrass (Cymbopogon flexuosus, C. citratus, C. pendulus and C. species). In Govil JN, Bhattacharya S, Eds., Recent Progress in Medicinal Plants: Essential Oils I. Vol. 36. Studium Press LLC, Houston, USA. pp. 213-257.

Rismanbaf A, Zarei S (2020) Liver and kidney injuries in COVID-19 and their effects on drug therapy; a letter to editor. Arch Acad Emerg Med 8(1):e17.

Singh DD (2018) Assessment of antimicrobial activity of hundreds extract of twenty Indian medicinal plants. Biomed Res 29(9):1797-1814.

Suresh A, Abraham J (2020) Phytochemicals and their role in pharmaceuticals. In Patra J, Shukla A, Das G, Eds., Advances in Pharmaceutical Biotechnology. Springer, Singapore. pp. 193-218.

Tripet B, Howard MW, Jobling M, Holmes RK, Holmes KV, Hodges RS (2004) Structural characterization of the SARS-coronavirus spike S fusion protein core. J Biol Chem 279(20):20836-20849.

Trott O, Olson AJ (2010) AutoDock Vina: improving the speed and accuracy of docking with a new scoring function, efficient optimization, and multithreading. J Comput Chem 31(2):455-461.

ul Qamar MT, Alqahtani SM, Alamri MA, Chen LL (2020) Structural basis of SARS-CoV-2 3CLpro and anti-COVID-19 drug discovery from medicinal plants. J Pharm Anal 10(4):313-319.

WHO Coronavirus Disease (COVID-19) Dashboard (https:// covid19.who.int/) [accessed 13 January 2021].

Walls AC, Park YJ, Tortorici MA, Wall A, McGuire AT, Veesler D (2020) Structure, function, and antigenicity of the SARS-CoV-2 spike glycoprotein. Cell 181(2):281-292.

Wang Q, Zhang Y, Wu L, Niu S, Song C, Zhang Z, Lu G, Qiao C, Hu Y, Yuen KY, Wang Q (2020) Structural and functional basis of SARS-CoV-2 entry by using human ACE2. Cell 81(4):894-904.

Waterhouse A, Bertoni M, Bienert S, Studer G, Tauriello G, Gumienny R, Heer FT, de Beer TA, Rempfer C, Bordoli L, Lepore R (2018) SWISS-MODEL: homology modelling of protein structures and complexes. Nucleic Acids Res 46(W1):W296-303.

Yan R, Zhang Y, Li Y, Xia L, Guo Y, Zhou Q (2020) Structural basis for the recognition of SARS-CoV-2 by full-length human ACE2. Science 367(6485):1444-1448.

Yan Y, Tao H, He J, Huang SY (2020) The HDOCK server for integrated protein-protein docking. Nat Protoc 15(5):1829-1852.

Yang XH, Deng W, Tong Z, Liu YX, Zhang LF, Zhu H, Gao H, Huang L, Liu YL, Ma CM, Xu YF (2007) Mice transgenic for human angiotensin-converting enzyme 2 provide a model for SARS coronavirus infection. Comp Med 57(5):450-459.

Yao X, Ye F, Zhang M, Cui C, Huang B, Niu P, Liu D (2020) In vitro antiviral activity and projection of optimized dosing design of hydroxychloroquine for the treatment of severe acute respiratory syndrome coronavirus 2 
(SARS-CoV-2). Clin Infect Dis 71(15):732-739.

Yazdany J, Kim AH (2020) Use of hydroxychloroquine and chloroquine during the COVID-19 pandemic: what every clinician should know. Ann Int Med 172(11):754-755.
Zhou P, Yang XL, Wang XG, Hu B, Zhang L, Zhang W, Si HR, Zhu Y, Li B, Huang CL, Chen HD (2020) A pneumonia outbreak associated with a new coronavirus of probable bat origin. Nature 579(7798):270-273. 
